• 研究报告・

\title{
榕小蜂温度耐受性及其对种间共存关系的影响
}

\author{
陈欢欢 ${ }^{1,2}$ 李竹梅 ${ }^{1}$ 唐利洲 ${ }^{*}$ \\ 1 (曲靖师范学院云南高原生物资源保护与利用研究中心/生物资源与食品工程学院/云南省高校云贵高原动植物多样性及 \\ 生态适应性进化重点实验室, 云南曲靖 655011) \\ 2 (中国科学院西双版纳热带植物园综合保护中心, 云南㐨腊 666303)
}

\begin{abstract}
摘要: 全球已知有约800种榕属(Ficus)植物, 主要分布在热带, 部分种类延伸至亚热带地区。温度是限制榕-蜂共生 系统分布北界的主要因素, 也显著影响榕树及其榕小蜂的繁殖成功, 其中榕小蜂对温度的响应更加敏感。榕小蜂 只有在一定的温度范围内才能保持正常的生理机能, 其对温度耐受能力直接影响榕果内小蜂种群数量和群落内种 间关系。然而目前对榕小蜂温度耐受性的研究尚少, 榕小蜂的温度耐受能力如何影响榕果内小蜂的共存关系还未 见报道。本文研究了分布于西双版纳的2种雌雄同株和1种雌雄异株榕树果内传粉榕小蜂和非传粉小蜂的温度耐受 能力。结果表明: 3 种传粉榕小蜂对高温的耐受性极差, 相对于雌雄同株的高榕 (F. altissima) 和聚果榕(F. racemosa) 传粉榕小蜂, 雌雄异株的鸡嗉子榕(F. semicordata) 传粉榕小蜂对低温有增强的耐受趋势。聚果榕小蜂群落结构显 示: 在适宜其生长的西双版纳地区, 传粉榕小蜂的数量占绝对优势, 在温度较低的季节其数量显著减少; 而非传 粉榕小蜂呈相反模式, 较强的温度耐受能力使其在低温的雾凉季维持了较高的种群数量。鸡嗉子榕果内非传粉小 蜂Sycoscapter trifemmensis相对于Philotrypesis dunia有更强的温度耐受能力, 在种群数量和种间关系上有更多的竞 争优势及数量。榕小蜂的温度耐受性差异在物种分布、种间关系的维持和共存上起了重要作用, 本研究结果为阐 明榕小蜂种间共存的维持机制提供了科学依据。
\end{abstract}

关键词: 榕小蜂; 生理特性; 温度耐受性; 群落动态

\section{Effects of fig wasp temperature tolerance on interspecific coexistence}

\author{
Huanhuan Chen ${ }^{1,2}$, Zhumei Li ${ }^{1}$, Lizhou Tang ${ }^{1 *}$ \\ 1 Center for Yunnan Plateau Biological Resources Protection and Utilization/College of Biological Resource and Food \\ Engineering/Key Laboratory of Yunnan Province University of the Diversity and Ecological Adaptive Evolution for \\ Animals and Plants on Yungui Plateau, Qujing Normal University, Qujing, Yunnan 655011 \\ 2 Center for Integrative Conservation, Xishuangbanna Tropical Botanical Garden, Chinese Academy of Sciences, \\ Mengla, Yunnan 666303
}

\begin{abstract}
There are about 800 species of fig trees in the world, which are mainly distributed in tropics, some species extend to subtropics. Fig trees rely on agaonid fig wasps to pollinate and are also exploited by some non-pollinating fig wasps. Fig wasps depend heavily on temperature who can only maintain normal physiological functions within a certain temperature range that is more sensitive than fig trees. Temperature directly affects the fig wasp's abundance and interspecific relationships. However, lack of research leaves a knowledge gap in our understanding of how fig wasp temperature tolerance affects the coexistence of wasps in fig. We conducted the experiment of temperature tolerance on six species of fig wasps from monecious Ficus altissima and F. racemosa, and dioecious F. semicordata in Xishuangbanna. Our results show that high temperature tolerance of all pollinating fig wasps was low, while the tolerance of Ceratosolen gravelyi on $F$. semicordata to low temperature was enhanced. When looking at fig wasp community composition on $F$. racemosa, the pollinating fig wasp are dominant species, but the population was decreased significantly in low temperature seasons in Xishuangbanna. While non-pollinating fig wasps showed the opposite pattern, which maintained high abundances during the cool-foggy season with low temperature due to greater
\end{abstract}


temperature tolerances. On F. semicordata, non-pollinating fig wasps Sycoscapter trifemmensis had stronger temperature tolerance than Philotrypesis dunia so that the former had competitive advantage and large population. Differences in temperature tolerance of fig wasps plays an important role in species distribution, maintaining interspecific relationships and coexistence. Our results provide scientific evidence for understanding fig wasp coexistence.

Key words: fig wasp; physiology; temperature tolerance; community dynamic

榕-蜂互惠系统稳定性维持机制一直是研究的 热点(Cruaud et al, 2012; Chen et al, 2015, 2018)。榕 树与榕小蜂之间稳定的互惠关系是长期进化的结 果。榕树与其专一的传粉榕小蜂在繁殖上相互依赖, 构成了生物界最为经典的互惠系统。除了传粉榕小 蜂, 榕果内还共生着1-32种不等的非传粉榕小蜂, 构成一个互惠、共存的榕-蜂微生态系统(Peng et al, 2010)。全球已知约800种榕属(Ficus)植物的分布局 限在热带、亚热带地区(Zhou \& Michael, 2003)。已 有的研究结果表明温度限制了榕-蜂互惠系统北界 的分布, 榕树及其榕小蜂的繁殖显著受温度影响, 其中榕小蜂对温度的响应更加敏感(Chen et al, 2018)。榕小蜂只有在一定的温度范围内才能保存正 常的生理机能(Jevanandam et al, 2013), 传粉榕小蜂 羽化后的存活时间对榕-蜂互惠系统的维持有重要 的影响。高温可导致榕小蜂死亡, 同时榕小蜂在低 温时会进入可恢复的昏迷状态, 但是这个温度对其 寄主榕树没有影响。非传粉榕小蜂有更强的生理耐 受能力, 因而比传粉榕小蜂有更大的分布范围, 前 人的研究发现它们可在无传粉榕小蜂的榕果中发 育(Kjellberg et al, 1987; Sutton et al, 2016)。

榕小蜂的温度耐受能力影响其出蜂后的产卵 时间, 进而影响榕小蜂在榕果内的种群数量, 且表 现出明显的季节性(Warren et al, 2010)。在西双版纳 热带地区, 季节性气候变异较大, 干季繁殖种子和 传粉榕小蜂更为成功, 雨季则繁殖更多的非传粉小 蜂(Peng et al, 2010)。在台北亚热带气候环境中, 小 叶榕(Ficus microcarpa)夏季结果量大, 传粉榕小蜂 种群也最大, 冬季传粉榕小蜂种群最小, 进入春季 后种群迅速恢复(Yang et al, 2013)。榕小蜂温度耐受 能力直接影响榕果内榕小蜂种群数量和群落内种 间关系, 适当的种群大小和错综复杂的种间关系在 维系这个共生系统平衡上起了关键的作用 (Kerdelhué et al, 2000; 谷海燕和杨大荣, 2003; Xu et al, 2003; Yadav \& Borges, 2018)。Warren等(2010)
用生态位模型研究了同时进入榕树Ficus sycomorus 榕果内的两种小蜂Ceratosolen arabicus和C. galili的 共存关系, 结果表明尽管二者的分布区存在重叠, 但由于传粉榕小蜂C. arabicus对温度有更高的生理 耐受能力从而在榕果内有更高的丰富度。West等 (1996)的研究发现榕果内非传粉小蜂的种群数量受 温度的影响, 温度同时影响非传粉榕小蜂之间的竞 争排斥关系。杨大荣等 $(2000,2001)$ 对聚果榕 $(F$. racemosa) 榕果内小蜂群落结构研究发现榕小蜂存 在明显的季节性变化。由此可见温度对小蜂群落构 成和小蜂共存有直接影响。但由于目前对榕小蜂本 身温度耐受性的研究尚少, 温度对榕果内的小蜂数 量及其共存关系的影响还未有报道, 截至目前, 仅 Jevanandam等(2013)报道过3种雌雄异株榕树Ficus grossularioides、F. fistulosa、F. variegata和1种雌雄 同株榕树F. microcarpa传粉榕小蜂对高温的耐受能 力, 有关传粉榕小蜂对低温的耐受性以及非传粉榕 小蜂的温度耐受能力目前则未见报道。相对于雌雄 同株的榕树, 雌雄异株榕树更适应分布北缘的环境, 有更广的分布范围(Bain et al, 2014), 常延伸到亚热 带地区。这种分布差异是否与不同起源榕树榕果内 榕小蜂温度耐受能力不同有关还未有报道。

西双版纳的榕树种类极其丰富, 这里自然分布 着81个种(32个雌雄同株, 49 个雌雄异株)。为探明 温度对该地区榕果内榕小蜂群落构成有何影响, 以 及传粉榕小蜂和非传粉榕小蜂在榕果内的共存关 系, 本研究选择自然分布于西双版纳的两种雌雄同 株榕树高榕(F. altissima)、聚果榕(F. racemosa) 以及 一种雌雄异株榕树鸡嗉子榕(F. semicordata), 对存 在于榕果内的传粉小蜂和非传粉小蜂进行温度耐 受性实验, 同时调查统计榕果内的小蜂群落动态, 以期回答如下问题: (1)传粉榕小蜂对温度的耐受能 力如何? (2)非传粉榕小蜂对温度的耐受能力如 何? (3)榕小蜂的生理耐受能力对榕小蜂群落有哪 些影响? 


\section{材料与方法}

\section{1 样地概况}

研究样地位于云南省西双版纳傣族自治州中 国科学院西双版纳热带植物园及其所在跁仑镇 $\left(101^{\circ} 15^{\prime} \mathrm{E}, 21^{\circ} 55^{\prime} \mathrm{N}\right)$ 周边地区, 样地的平均海拔约 $600 \mathrm{~m}$ 。西双版纳位于东南亚热带北缘, 干旱季分明, 降雨主要集中在雨季(5-10月), 旱季为3-4月, 雾凉 季从11月到次年2月。属于典型的热带季风型气候, 年平均气温 $21.8^{\circ} \mathrm{C}$, 年平均相对湿度 $86 \%$, 年降雨 量为 $1,557 \mathrm{~mm}$ 。其中1月份为最冷月份, 平均气温 $11.2^{\circ} \mathrm{C}, 4$ 月份最热, 平均气温为 $33.5^{\circ} \mathrm{C}$ 。图1为实验 期间的温度图。

\section{2 研究材料}

高榕为雌雄同株, 是桑科榕属榕亚属(subgen. Urostigma)的高大乔木, 主要分布于印度至中国南 部、缅甸、印度支那半岛、泰国、印度尼西亚等地 区(Zhou \& Michael, 2003)。单株树一年内可多次结
发育经历5 个时期, 即䧳花前期(A期)、雌花期(B 期)、间花期 $(\mathrm{C}$ 期 $) 、$ 雄花期 $(\mathrm{D}$ 期)和花后期 $(\mathrm{E}$ 期 $)$ 。传 粉榕小蜂在雌花期进入榕果内产卵, 雄花期从榕果 内羽化后出蜂, 继续寻找雌花期榕果。非传粉榕小 蜂在果外产卵, 羽化时间与传粉榕小蜂相同。高榕 的传粉榕小蜂为Eupristina altissima, 高榕榕果内的 非传粉榕小蜂丰富, 多达32种。

聚果榕为雌雄同株, 隶属于聚果榕亚属 (subgen. Sycomorus)。树高可达30 m, 高大乔木, 树

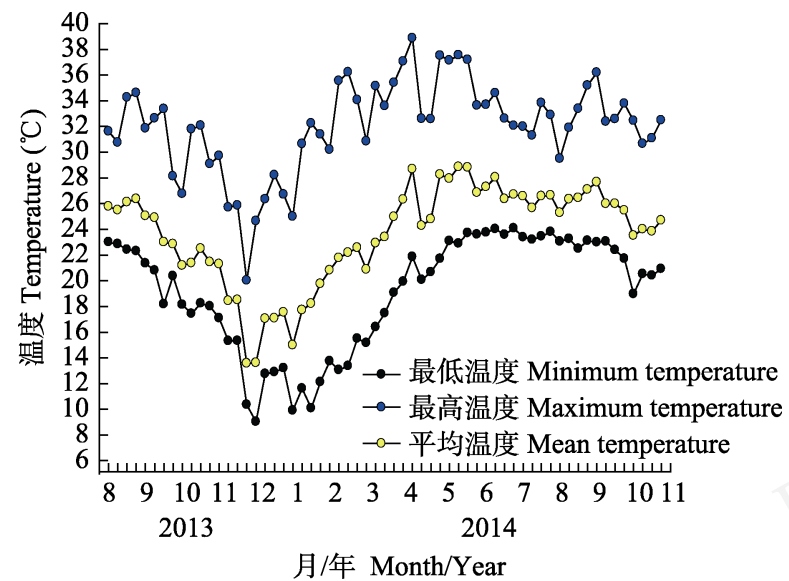

图1 实验期间的最低温度、最高温度和平均温度

Fig. 1 Minimum temperature, maximum temperature and average temperature in the study site at Xishaungbanna during the period of the study
干结果。分布于亚洲热带的大部分地区。其传粉者 为 Ceratosolen sp., 为主动传粉类型。聚果榕中的非 传粉榕小蜂包括 Sycophaga testacea, S. mayri, S. agraensis, Apocrypta westwoodi 和 Apocrypta sp. 等 5 种。

鸡嗉子榕为雌雄异株, 隶属于聚果榕亚属 (subgen. Sycomorus)。树高可达15 m。广泛分布于亚 洲热带地区。榕果生于老茎上，果枝下垂至根部或 穿入土中，常见于海拔600-1,600 m的路旁、林缘或 沟谷。鸡嗉子榕榕树每年大约能结2-3批果，一般在 温暖季节结果，树内榕果同步而树间异步。鸡嗉果 的传粉榕小蜂为Ceratosolen gravelyi, 与之共存的 还有 4 种非传粉榕小蜂, 包括 Sycophaga cunia, Sycoscapter trifemmensis, Philotrypesis dunia 和 Apocrypta sp.。

\section{3 研究方法}

\subsection{1 榕小蜂生理耐受实验}

分别选择3株进入雄花期的高榕、聚果榕和鸡 嗉子榕树，高榕每株随机采集30个榕果，聚果榕、 鸡嗉子榕每株随机采集 20 个榕果。分装在 120 目的 纱网隔离袋内, 让果内榕小蜂在室温下自然羽化 $1.5 \mathrm{~h}$ 进入袋中, 以保证榕小蜂处于相同的年龄阶段 以及减少榕小蜂对环境的适应。将羽化后的榕小蜂 分装在不同的纱网袋中, 每种小蜂的个体数在 10 个 以上。该实验在Grant GRD1-LH多室温度梯度培养 箱中进行, 温度从 $10-36^{\circ} \mathrm{C}$ 每个梯度温度以 $2^{\circ} \mathrm{C} \pm$ $0.5^{\circ} \mathrm{C}$ 递增, 每个梯度下置以 PDA培养基以保证培 养箱内的相对湿度。将分装好的榕小蜂分别放置于 不同的温度梯度室内, 每 2 h 记录每个温度梯度下 榕小蜂的存活情况直至榕小蜂死亡或进入不可逆 转的不能移动状态。在温度为 $36^{\circ} \mathrm{C}$ 时, 每小时对榕 小蜂的存活情况进行记录。

\subsection{2 自然果收集榕小蜂}

从2013年8月到2014年11月对西双版纳的高榕、 聚果榕及鸡嗉子榕的物候进行定树、定时系统观测, 每周观察1次。其中高榕12株, 聚果榕23株, 鸡嗉子 榕雄树 14 株。两株树间的距离从数米到数千米。每 次观测中，发现树上有雄花期的果就及时采摘，每 棵树上随机采 30 个榕果, 每一批榕果的采集在榕果 达到 $\mathrm{D}$ 期时的 3 天内完成。采集的榕果带回实验室单 果分装在120篮目 $(20 \mathrm{~cm} \times 20 \mathrm{~cm})$ 的绢纱网中让其自 然羽化出蜂, 最后收集每个榕果内羽化出来的榕小 
蜂, 置于 $75 \%$ 的酒精中保存。在实验室内借助Leica S8APO体视显微镜, 对高榕、聚果榕以及鸡嗉子榕 榕果内的传粉榕小蜂和非传粉榕小蜂进行分类鉴 定, 并统计每种蜂的个体数量。

\section{3 结果}

\subsection{3 种传粉榕小蜂的存活时间}

持续记录3种传粉榕小蜂的存活时间, 结果表 明(图2): 在温度达到 $36^{\circ} \mathrm{C}$ 时, 3 种传粉榕小蜂的存 活时间仅有 $4 \mathrm{~h}$, 温度高于 $30^{\circ} \mathrm{C}$ 时, 3 种传粉榕小蜂 的寿命均小于 $24 \mathrm{~h}$ 。而当温度低于 $30^{\circ} \mathrm{C}$ 时, 高榕传 粉榕小蜂的存活时间显著增加, 温度为 $28^{\circ} \mathrm{C}$ 时, 其 存活时间已达 $44 \mathrm{~h}$, 在温度为 $16^{\circ} \mathrm{C}$ 时可达 $150 \mathrm{~h}$ 之 久。之后随着温度的继续降低, 高榕传粉榕小蜂的 存活时间有所降低, 但仍有较长的存活时间。与之 相比，聚果榕和鸡嗉子榕传粉榕小蜂的存活时间在 14-30 ${ }^{\circ} \mathrm{C}$ 之间随着温度降低逐步增加, 在温度为 $14^{\circ} \mathrm{C}$ 时二者的存活时间分别为 $46 \mathrm{~h}$ 和 $44 \mathrm{~h}$ 。随着温 度继续降低, 聚果榕和鸡嗉子榕传粉榕小蜂的存活 时间显著增加, 在 $10^{\circ} \mathrm{C}$ 时分别为 $94 \mathrm{~h}$ 和 $124 \mathrm{~h}$, 其中 鸡嗉子榕传粉榕小蜂的存活时间增加更为明显。

\section{2 聚果榕榕小蜂的存活时间}

持续记录聚果榕中的两种非传粉榕小蜂的存 活时间, 结果显示(图3): 在温度大于 $34^{\circ} \mathrm{C}$ 时, 两种 非传粉榕小蜂的寿命为 $16 \mathrm{~h}$; 当温度在 $24-34^{\circ} \mathrm{C}$ 时, Sycophaga mayri比S. testacea 有较长的存活时间, 温 度为 $24^{\circ} \mathrm{C}$ 时, S. mayri和S. testacea的存活时间分别

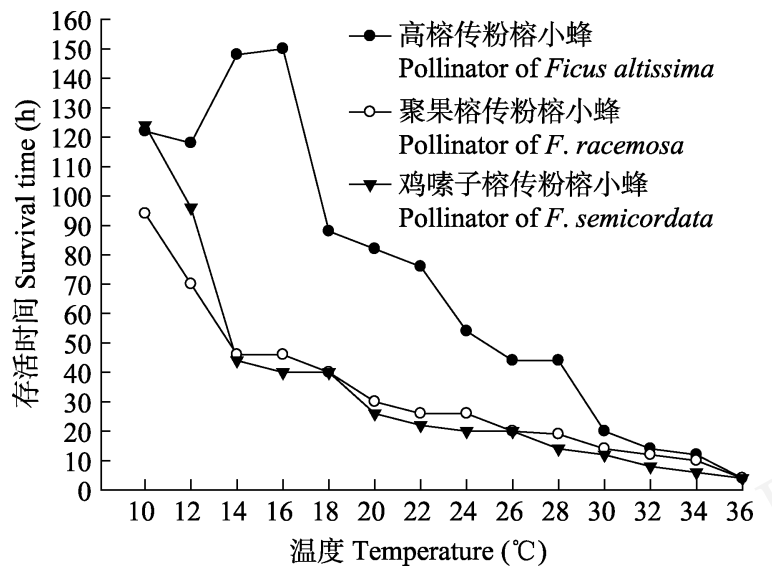

图2 高榕、聚果榕、鸡嗉子榕中传粉榕小蜂在不同温度下 的存活时间

Fig. 2 Pollinator lifespan of Ficus altissima, F. racemosa and F. semicordata at different temperature
为 $76 \mathrm{~h}$ 和 $70 \mathrm{~h}$ 。当温度低于 $24^{\circ} \mathrm{C}$ 时, S. testacea 的存活 时间则较S. mayri更长, 两者的存活时间在 $16^{\circ} \mathrm{C}$ 时 均达到最长, S. mayri和S. testacea的存活时间分别 能达到 $150 \mathrm{~h}$ 和 $186 \mathrm{~h}$ 。当温度为 $10^{\circ} \mathrm{C}$ 时，两种小蜂处 于可恢复的不移动状态，随着低温的持续，榕小蜂 则变得丧失生理机能。S. mayri和S. testacea在温度 为 $10^{\circ} \mathrm{C}$ 时的存活时间分别为 $136 \mathrm{~h}$ 和 $178 \mathrm{~h}$ (图3)。

\section{3 鸡嗉子榕榕小蜂的存活时间}

持续记录鸡嗉子榕中的两种非传粉榕小蜂的 存活时间, 结果显示(图4): 当温度达到 $36^{\circ} \mathrm{C}$ 时, $S$. trifemmensis与 $P$. dunia的存活时间分别为 $26 \mathrm{~h}$ 和 $22 \mathrm{~h}$; 在温度为 $30^{\circ} \mathrm{C}$ 时，两者的存活时间延长至36 h 和 26 $\mathrm{h}$; 在温度为 $24^{\circ} \mathrm{C}$ 时, S. trifemmensis与 $P$. dunia的存 活时间分别为 $64 \mathrm{~h}$ 和 $56 \mathrm{~h}$ 。当温度低于 $24^{\circ} \mathrm{C}$ 时, $S$. trifemmensis的存活时间比P. dunia增加更为明显。随 着温度继续降低，两种非传粉榕小蜂的存活时间有 显著增加的趋势。S. trifemmensis的存活时间在 $10^{\circ} \mathrm{C}$ 时已高达 294 h, P. dunia的存活时间达 224 h (图4)。

\subsection{3 种榕树榕果内小蜂群落结构特征}

共采集高榕榕果183个，收集传粉榕小蜂 21,131只。传粉榕小蜂羽化成熟集中在温度较低的 月份，1-3月单个榕果内传粉榕小蜂平均为 91.1 、 133.7、169.8只。4-6月榕果内未发现传粉榕小蜂, 7、 8、9、11月份, 传粉榕小蜂重新出现, 单果内传粉 榕小蜂数量平均为81.4、180、209.4、39.6只。

共采集聚果榕榕果173个, 收集传粉榕小蜂 133,009只。样树上除了1月份没有收集到传粉榕小 蜂外，其余月份均有传粉榕小蜂活动。4-9月单果传

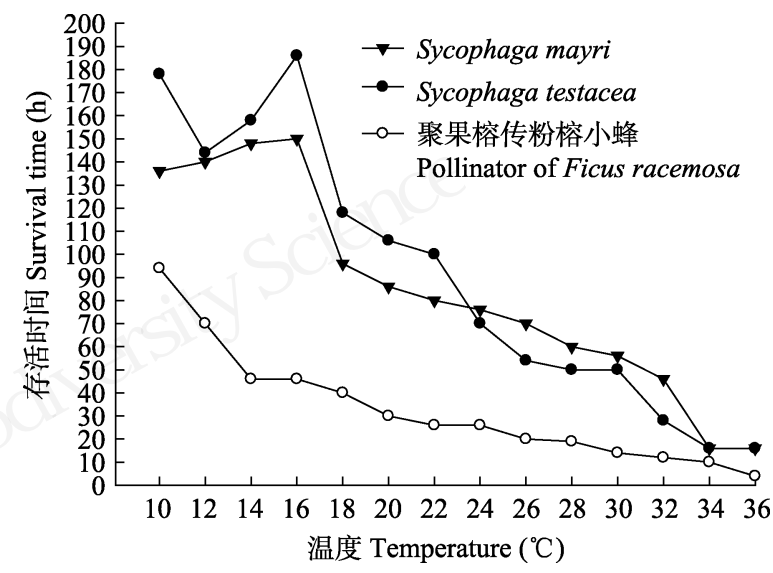

图3 聚果榕榕小蜂在不同温度下的存活时间

Fig. 3 Fig wasp lifespan of Ficus racemosa at different temperature 


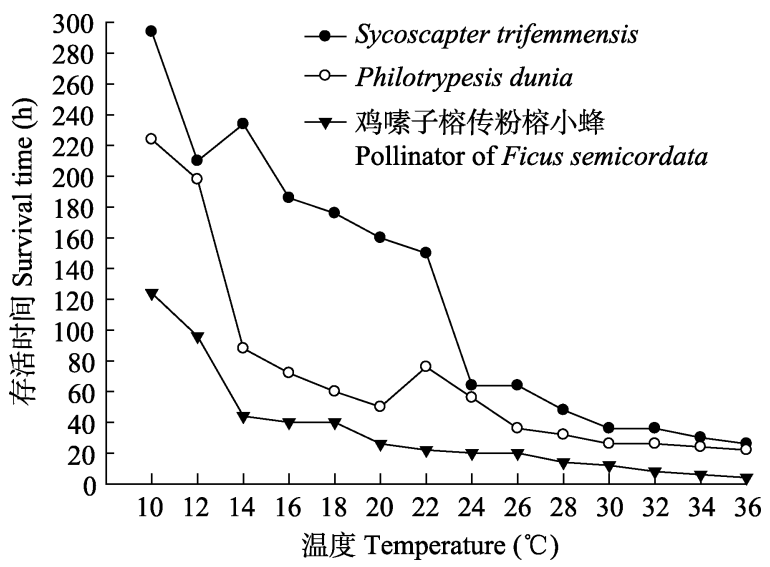

图4 鸡嗉子榕榕小蜂在不同温度下的存活时间

Fig. 4 Fig wasp lifespan of Ficus semicordata at different temperature
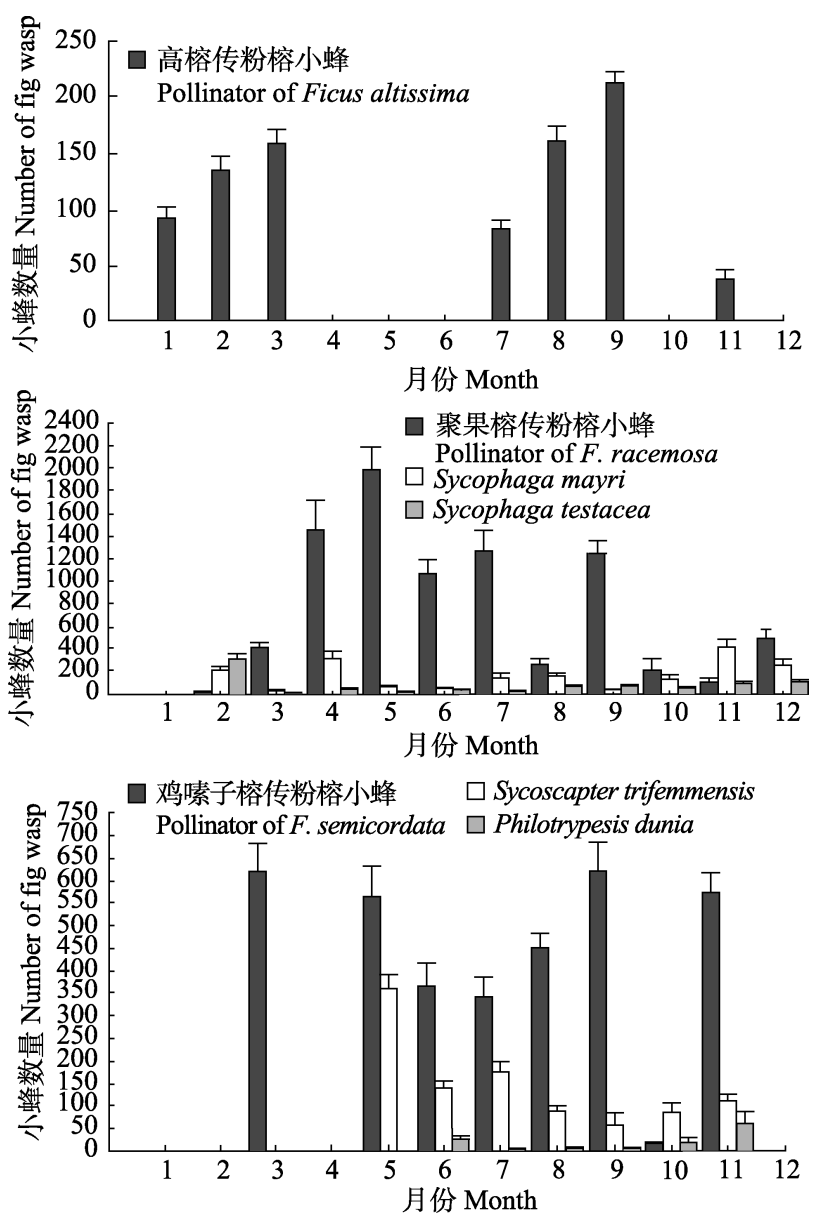

图5 高榕、聚果榕、鸡嗉子榕榕果内小蜂群落结构

Fig. 5 Fig wasp community structure of Ficus altissima, F. racemosa and F. semicordata

粉榕小蜂平均数量较多, 分别为 $1,434.9 、 1,854.9$ 、 $1,067.4 、 1,274.9 、 222.6 、 1,265.4$ 只。在温度较低的 雾凉季和干旱季(10、11、12、2、3月), 聚果榕单果
内传粉榕小蜂的平均数量较少, 分别为217.5、86.6、 518.7、8.3、404.9只。

共采集鸡嗉子榕榕果136个, 收集传粉榕小蜂 60,171只。其中1、2、4月及12月未采集到传粉榕小 蜂，3月及 5-11月单果内平均传粉榕小蜂数量为 621.7、555.7、359.3、332.2、448.8、621.0、16.6、 519.8只。其中在温度较低的3月和11月, 榕果内传 粉榕小蜂的平均数量也较大(图5)。

收集的鸡嗉子榕榕果内, S. trifemmensis 有 16,623只, P. dunia 1,734只。其中6-11月, 榕果内这 两种非传粉榕小蜂同时出现, 单果内S. trifemmensis 的平均数量显著大于 $P . d u n i a$ 。采集的聚果榕榕果内, S. mayri有25,927只, S. testacea有10,842只。样树上 除了1月没有采集到两种小蜂外，其余月份榕果内 两种非传粉榕小蜂均同时出现, 单果内S. mayri的 平均数量多于S. testaca (图5)。

\section{4 讨论}

榕树的分布局限在热带、亚热带地区。温度是 其地理分布的主要限制因素(Chen et al, 2018), 相 对于榕树, 榕小蜂对温度更为敏感。榕-蜂互惠系统 的成功繁殖很大程度上取决于传粉榕小蜂的温度 耐受能力。研究不同温度下高榕、聚果榕、鸡嗉子 榕3种传粉榕小蜂的存活时间, 结果显示传粉榕小 蜂对高温的生理耐受性极差, 当温度升高到 $36^{\circ} \mathrm{C}$ 时, 3 种传粉榕小蜂的存活时间仅有 $4 \mathrm{~h}$ 。温度高于 $30^{\circ} \mathrm{C}$ 时, 3 种榕小蜂的存活时间均小于 $24 \mathrm{~h}$, 该结果与 Jevanandam等(2013)对新加坡4种传粉榕小蜂温度 耐受性的研究结果相一致。温度低于 $14^{\circ} \mathrm{C}$ 时, 随着 温度的降低，鸡嗉子榕传粉榕小蜂的存活时间相较 于聚果榕传粉榕小蜂明显增加, 而高榕的传粉榕小 蜂在温度低于 $16^{\circ} \mathrm{C}$ 时，存活时间有下降的趋势。高 榕和聚果榕是典型的热带分布种，鸡嗉子榕广泛分 布于亚热带地区(Berg \& Corner, 2005) , 不同物种 的分布差异反映在传粉榕小蜂生理耐受性上，可能 与其对低温的生理耐受能力有关，正如研究结果所 显示, 鸡嗉子榕的传粉榕小蜂对低温有增强的生理 耐受趋势。该结果也与鸡嗉子榕有更广的分布相吻 合。而同为热带分布种，高榕上的传粉榕小蜂相对 于聚果榕传粉榕小蜂有极长的存活时间，早期的研 究发现在高榕上存在罕见的小蜂等待现象(Suleman 
et al, 2011; Zhang et al, 2014), 即在榕果进入接收期 之前, 高榕叶片上发现成千上万提前到达榕果的传 粉榕小蜂。这一现象的出现可能与榕树与榕小蜂对 气候变化的响应不同步有关, 以至于在繁殖衔接上 出现了问题, 而高榕传粉榕小蜂进化出极长的存活 时间则保证了其繁殖的成功。

西双版纳位于亚洲热带北缘, 季节性气候变化 明显。该区有大量的聚果榕分布, 并且温度也适合 榕小蜂生存, 聚果榕榕果内传粉榕小蜂和非传粉榕 小蜂同时存在(Wang \& Sun, 2009)。由于温度耐受能 力的差异, 传粉榕小蜂数量占绝对优势。向北延伸 到亚热带的六库地区, 随着温度降低, 榕果内的传 粉榕小蜂丰富度明显降低, 而能耐受更低温度的非 传粉榕小蜂则保持了较大的种群数量(Chen et al, 2018)。由于非传粉榕小蜂一般是在果外产卵, 受温 度影响更大, 因此进化出更强的温度耐受性(Peng et al, 2010)。研究结果表明, 聚果榕内传粉榕小蜂的 数量在温度较低的 10 月到次年 3 月份数量显著减少, 而两种非传粉榕小蜂S. mayri和S. testacea的种群数 量则恰恰相反, 在温度较低的月份种群数量相对较 大。产生这种季节性差异的原因是由于传粉榕小蜂 在温度低于 $10^{\circ} \mathrm{C}$ 时已处于可恢复的不移动状态, 而 非传粉榕小蜂由于更强的温度耐受性则维持了较 大的种群数量。这一结果也与非传粉榕小蜂有更广 的分布相一致：例如F. carica在其自然分布区法国 蒙彼利埃没有传粉榕小蜂(Kjellberg et al, 1987), 此 外, 还有大量被种植于自然分布区外的榕树种没有 发现传粉榕小蜂, 但却有1-2种非传粉榕小蜂(Lin et al, 2008)。

与聚果榕类似, 鸡嗉子榕榕果内传粉榕小蜂和 非传粉榕小蜂也呈现出明显的季节性, 且种群间的 数量差异较大。共存于榕果内的传粉榕小蜂数量较 多, 而非传粉榕小蜂种群数量较少。在西双版纳地 区, 鸡嗉子榕榕果内的小蜂羽化出蜂集中在温度较 高的月份, 除了 5 月外, 两种非传粉小蜂 $S$. trifemmensis和P. dunia共存于榕果, 且S. trifemmensis的数量显著大于 $P$. dunia。这一结果与鸡嗉子小蜂 群落中S. trifemmensis有更大的种群数量相一致(王 秋艳等, 2003; 宋波等, 2008)。榕果内小蜂适度的种 群数量和种间关系对这个共生系统的维持至关重 要(Yadav \& Borges, 2018), 榕小蜂的温度耐受能力
在种间关系维持上起了关键作用, 直接影响了榕果 内小蜂的数量。榕小蜂温度耐受性研究结果显示, $S$. trifemmensis有更强的温度耐受能力, 保证了其在果 外有更长的产卵时间。种间竞争关系的存在对榕果 内小蜂群落的稳定共存起了重要作用(Warren et al, 2010), 榕小蜂自身通过长期进化而具有的不同的 温度耐受能力是种间关系发挥作用的基础。鸡嗉子 榕榕果内温度耐受能力较强的S. trifemmensis有更 多的种群数量, 不同属的小蜂P. dunia由于相对较 低的温度耐受性在种间竞争上表现出劣势。

榕小蜂羽化出蜂后的存活时间对榕小蜂的飞 行距离以及能否找到接收期的榕果并完成传粉有 重要影响(Warren et al, 2010), 而榕小蜂的存活时间 取决于其温度耐受性, 由此, 榕小蜂的温度耐受性 与榕树的分布直接相关。榕小蜂自身的温度耐受性 也是其与环境及其寄主榕树之间长期进化的结果。 从榕树与榕小蜂系统发育的角度出发, 结合榕小蜂 的温度耐受数据, 可进一步探讨不同种榕树中榕小 蜂与榕树的起源演化。目前榕树分布模型是基于榕 树本身对环境的适应性进行拟合的，榕树与榕小蜂 作为专性互惠系统, 榕小蜂生理耐受数据的缺乏使 得榕树分布模拟的工作受到很大的限制。我们的实 验结果揭示了传粉榕小蜂对低温和高温的耐受能 力以及不同种榕树中非传粉榕小蜂的温度耐受性, 更多榕小蜂生理耐受性实验可为榕树分布的模拟 以及榕-蜂互惠系统对气候变化的响应提供依据, 对进一步阐明在全球气候变化过程中物种共存关 系及榕树分布区的变化提供有力的支撑。

致谢: 感谢中国科学院昆明植物研究所中国西南野 生生物种质资源库郭云刚老师在实验中提供设备 帮助。

\section{参考文献}

Bain A, Chou LS, Tzeng HY, Ho YC, Chiang YP, Chen WH, Chio YT, Li GY, Yang HW, Kjellberg F, Hossaert-McKey M (2014) Plasticity and diversity of the phenology of dioecious Ficus species in Taiwan. Acta Oecologica, 57, 124-134.

Chen HH, Peng YQ, Zhang Y, Corlett RT (2015) Winter cropping in Ficus tinctoria: An alternative strategy. Scientific Reports, 5, 16496.

Chen HH, Zhang Y, Peng YQ, Corlett RT (2018) Latitudinal 
effects on phenology near the northern limit of figs in China. Scientific Reports, 8, 4320.

Cruaud A, Rønsted N, Chantarasuwan B, Chou LS, Clement WL, Couloux A, Cousins B, Genson G, Harrison RD, Hanson PE, Hossaert-Mckey M, Jabbour-Zahab R, Jousselin E, Kerdelhué C, Kjellberg F, Lopez-Vaamonde C, Peebles J, Peng YQ, Pereira RAS, Schramm T, Ubaidillah R, van Noort S, Weiblen GD, Yang DR, Yodpinyanee A, Libeskind-Hadas R, Cook JM, Rasplus JY, Savolainen V (2012) An extreme case of plant insect co-diversification: Figs and fig-pollinating wasps. Systematic Biology, 61, 1029-1047.

Gu HY, Yang DR (2003) Community structure and species diversity of fig wasps from Ficus altissima. Biodiversity Science, 11, 188-196. (in Chinese with English abstract) [谷 海燕, 杨大荣 (2003) 高榕小蜂群落结构及物种多样性 的初步研究. 生物多样性, 11, 188-196.]

Jevanandam N, Goh AGR, Corlett RT (2013) Climate warming and the potential extinction of fig wasps, the obligate pollinators of figs. Biology Letters, 9, 20130041.

Kerdelhué C, Rossi JP, Rasplus JY (2000) Comparative community ecology studies on old world figs and fig wasps. Ecology, 81, 2832-2849.

Kjellberg F, Gouyon PH, Ibrahim M, Raymond M, Valdeyron G (1987) The stability of the symbiosis between dioecious figs and their pollinators: A study of Ficus carica L. and Blastophaga psenes L. Evolution, 41, 693-704.

Lin SL, Zhao NX, Chen YZ (2008) Phenology and the production of seeds and wasps in Ficus microcarpa in Guangzhou, China. Symbiosis, 48, 101-105.

Peng YQ, Yang DR, Compton SG (2010) The reproductive success of Ficus altissima and its pollinator in a strongly seasonal environment: Xishuangbanna, Southwestern China. Plant Ecology, 209, 227-236.

Song B, Peng YQ, Guan JM, Yang P, Yang DR (2008) Sex ratio adjustment of a non-pollinating fig wasp species on Ficus semicordata in Xishuangbanna. Chinese Journal of Applied Ecology, 19, 588-592. (in Chinese with English abstract) [宋波, 彭艳琼, 管俊明, 杨培, 杨大荣 (2008) 西双版纳鸡嗉果榕内一种非传粉小蜂的性比调节. 应用 生态学报, 19, 588-592.]

Suleman N, Raja S, Zhang Y, Compton SG (2011) Sexual differences in the attractiveness of figs to pollinators: Females stay attractive for longer. Ecological Entomology, 36, 417-424.

Sutton TL, Riegler M, Cook JM (2016) One step ahead: A parasitoid disperses farther and forms a wider geographic population than its fig wasp host. Molecular Ecology, 25, 882-894.

Wang QY, Yang DR, Peng YQ (2003) Pollination behaviour and propagation of pollinator wasps on Ficus semicordata in Xishuangbanna, China. Acta Entomologica Sinica, 46, 27-34. (in Chinese with English abstract) [王秋艳, 杨大荣, 彭艳琼 (2003) 西双版纳鸡嗉果榕小蜂繁殖和传粉行为. 昆虫学报, 46, 27-34.]

Wang RW, Sun BF (2009) Seasonal change in the structure of fig-wasp community and its implication for conservation. Symbiosis, 47, 77-83.

Warren M, Robertson MP, Greeff JM (2010) A comparative approach to understanding factors limiting abundance patterns and distributions in a fig tree-fig wasp mutualism. Ecography, 33, 148-158.

West SA, Herre EA, Windsor DM, Green PRS (1996) The ecology and evolution of the New World non-pollinating fig wasp communities. Journal of Biogeography, 23, 447-458.

Xu L, Yang DR, Peng YQ, Wang QY, Zhang GM (2003) The community structure and the interspecific relationship of the fig wasps in syconia of Ficus racemosa L. in Xishuangbanna, China. Acta Ecologica Sinica, 23, 1554-1560. (in Chinese with English abstract) [徐否, 杨大荣, 彭艳琼, 王 秋艳, 张光明 (2003) 西双版纳聚果榕隐头果内小蜂群 落结构及种间关系. 生态学报, 23, 1554-1560.]

Yadav P, Borges RM (2018) Host-parasitoid development and survival strategies in a non-pollinating fig wasp community. Acta Oecologica, 90, 60-68.

Yang DR, Wang RW, Song QS, Zhang GM, Zhao TZ (2000) Rule of seasonal changes of the Ceratosolen sp. in the tropical rainforest of Xishuangbanna, China. Forest Research, 13, 477-484.

Yang DR, Zhao TZ, Wang RW, Zhang GM, Song QS (2001) Study on pollination ecology of fig wasp (Ceratosolen sp.) in the tropical rainforest of Xishuangbanna, China. Zoological Research, 20, 125-130. (in Chinese with English abstract) [杨大荣, 赵庭周, 王瑞武, 张光明, 宋启示 (2001) 西双版纳热带雨林聚果榕小蜂的传粉生态学. 动 物学研究, 20, 125-130.]

Yang HW, Tzeng HY, Chou LS (2013) Phenology and pollinating wasp dynamics of Ficus microcarpa L. f.: Adaptation to seasonality. Botanical Studies, 54, 11.

Zhang Y, Peng YQ, Compton SG, Yang DR (2014) Premature attraction of pollinators to inaccessible figs of Ficus altissima: A search for ecological and evolutionary consequences. PLoS ONE, 9, e86735.

Zhou ZK, Michael GG (2003) Ficus through Moraceae. In: Flora of China, Vol. 5 (eds Wu ZY, Peter HR, Hong DY), pp. 37-71. Science Press, Beijing \& Missouri Botanical Garden Press, St. Louis.

(责任编委：朱朝东 责任编辑：时意专) 